\title{
The effect of peri-intraventricular hemorrhage on the auditory pathway of infants
}

\author{
Leticia Saia da Silva ${ }^{\mathrm{a}}$, Georgea Espindola Ribeiro ${ }^{\mathrm{b}}$, Jair Cortez Montovani ${ }^{\mathrm{c}}$, \\ Daniela Polo Camargo da Silva ${ }^{\mathrm{b}, \mathrm{d}, *}$ \\ a Speech Therapy, São Paulo State University (UNESP), Brazil \\ b General Surgery Program, Botucatu Medical School, São Paulo State University (UNESP), Brazil \\ ${ }^{c}$ Department of Ophthalmology, Otorhinolaryngology and Head and Neck Surgery, Botucatu Medical School, São Paulo State University (UNESP), Brazil \\ d Department of Speech-Language Pathology and Audiology, Federal University of Santa Catarina (UFSC), Brazil
}

\section{A R T I C L E I N F O}

\section{Keywords:}

Newborn

Hearing loss

Electrophysiology

Neonatal screening

\begin{abstract}
A B S T R A C T
Objective: To verify the effect of peri-intraventricular hemorrhage on the auditory pathway of preterm infants. Method: It is a non-concurrent cohort study. This study was conducted in a tertiary public. Preterm infants with peri-intraventricular hemorrhage comprised the study group, and preterm infants without peri-intraventricular hemorrhage were included as a comparison group, both were similar in relation to gestational age and risk indicators for hearing loss. Participants had to meet the following inclusion criteria: have been born at the study site, presence of otoacoustic emissions by transient stimulus in both ears and brainstem auditory evoked potentials with all components bilaterally identified.

Results: 44 infants with an average age of 3 months with peri-intraventricular hemorrhage and 2,6 months without peri-intraventricular hemorrhage met the inclusion criteria. Regarding the brainstem auditory evoked potentials results, a significant increase was observed in absolute latency values of waves I, III and V, as well as in the interpeak intervals I-III and I-V, bilaterally, in infants with peri-intraventricular hemorrhage.

Conclusion: This study concluded that infants with peri-intraventricular hemorrhage presented a delay in the neural conduction of sound, which justifies the monitoring of the auditory function in these infants during the period of language development.
\end{abstract}

\section{Introduction}

Intracranial hemorrhage is the major neurological pathology in newborns. Its most common manifestation is the peri-intraventricular hemorrhage (PIVH), followed by subdural, subarachnoid and cerebellar hemorrhage, which are less frequent [1].

$\mathrm{PIVH}$ is almost exclusively in preterm infants, especially those weighing below $1500 \mathrm{~g}$ at birth and with gestational age below 32 weeks, who are particularly more vulnerable to ischemic and hemorrhagic processes in the neonatal period, and which is closely related to a multifactor lesion of the germinative matrix [2-4].

Its diagnosis is basically performed through ultrasound of the skull still in the first days of life [5]. PIVH is classified according to the degree of severity from I to IV [6]. Grade I occurs when subependymal hemorrhage is restricted to the germinative matrix, in grade II there is intraventricular hemorrhage without ventricular dilatation, in grade III there is ventricular dilatation and in grade IV there is periventricular hemorrhagic infarction [7,8]. Grades III and IV are considered determinants for a poor prognosis [5].

However, the literature reports that those infants with grades between I and II may also present neurological abnormality, delayed in cognitive development and hearing impairment when compared with preterm infants and normal ultrasound [5].

Studies show that $7.4 \%$ of infants with PIVH present some degree of hearing impairment [9], this occurrence can be justified due to the fact that PIVH causes the impairment of the germinative matrix, a highly vascularized region located between the caudate nucleus and thalamus, from which glial cells and neurons responsible for myelination and cortical and subcortical development arise. Thus, PIVH can compromise the development and functioning of several structures, including the auditory, which shows the importance of early audiological assessment in these individuals [10].

In the neonatal population, electroacoustic and electrophysiological exams, such as the transient-evoked otoacoustic emission (TEOAE) and

\footnotetext{
* Corresponding author. Department of Speech-Language Pathology and Audiology, Federal University of Santa Catarina (UFSC), Campus Universitário Trindade s/n, 88040-900, Brazil.

E-mail address: daniela-polo@uol.com.br (D.P.C.d. Silva).
} 
brainstem auditory evoked potential (BAEP) are the most common technique for hearing screening. The first examination allows to verify, in detail, the functioning of the outer hair cells of the cochlea, the peripheral portion of the auditory system, and the second one predominantly examines the auditory pathway at the brainstem level [11].

Therefore, the hearing assessment in newborns with PIVH is necessary for a better understanding of the manifestations of this condition on the auditory pathway, which consequently may impair the development of hearing and language skills [12-14].

Thus, the aim of this study was to verify the effect of PIVH on the auditory pathway of preterm infants.

\section{Method}

This study was approved by the Research Ethics Committee of the institution (process number 2.206.522). It was a single non-concurrent cohort study, conducted from March to October 2017.

\subsection{Subjects}

Preterm infants with PIVH were included in the sample, composing the study group and preterm infants without PIVH composed the comparison group. The diagnosis of PIVH exposure was based on the result of the transfontanelar ultrasonography verified by the neonatal team of the maternity hospital $[7,15]$. Both groups were similar in relation to gestational age and risk indicators for hearing loss, according to the Joint Committee on Infant Hearing (JCIH) [16].

Inclusion criteria: have been born at the study site, presence of TEOAE in both ears, BAEP with all components bilaterally identified and having performed all auditory tests in the same session and Informed Consent signed by the parents.

Exclusion criteria: alterations in the external and/or middle ear detected by the otorhinolaryngologist, infants with genetic syndromes or craniofacial malformations.

\subsection{Outcome}

The main parameters evaluated in the BAEP of both groups were: absolute latency values of the waves I, III and V, and interpeak latency values of I-III, III-V and I-V in both ears.

\subsection{BAEP technical specifications}

The BAEP test was performed with the infants in natural sleep, using the equipment EP15 - Eclipse, Interacoustics /Denmark. After cleaning the skin with abrasive substance (Nuprep ${ }^{\circ}$ ), the surface electrodes byNeuroline ${ }^{\circ}$ were fixed at specific points. The active electrode was placed on the forehead (Fz) and the reference electrodes on the mastoid regions (M1 and M2). The ground electrode was placed on the forehead and the stimulus was presented through the ER 3A insertion earphone, with monaural stimulation with filtered clicks (high pass filter of $100 \mathrm{~Hz}$ and low pass filter of $2000 \mathrm{~Hz}$ ), duration of $100 \mu$ s and rarefied polarity. A total of 1024 clicks with analysis time of $20 \mathrm{~ms}$ were provided, repeated to confirm the reproducibility of the waves. The impedance of the electrodes was maintained below 3 Kohms. The stimulus presentation rate was 20.1 clicks per second and $80 \mathrm{dBnHL}$ intensity was used.

\subsection{Statistical analysis}

The comparison in relation to the potential confounders and the association of the absolute and interpeak latencies of the BAEP with PIVH was carried out through the non-parametric Mann-Whitney test and Chi-square test. The similarity of age between the groups was confirmed through Student's t-test. Differences were considered statistically significant when $\mathrm{p}<0.05$. The analysis was performed with the
Table 1

Sample characterization.

\begin{tabular}{lll}
\hline Groups/Variables & without PIVH $(\mathrm{n}=26)$ & with PIVH $(\mathrm{n}=18)$ \\
\hline Female & $13(50 \%)$ & $11(61 \%)$ \\
Male & $13(50 \%)$ & $7(39 \%)$ \\
Gestational age (weeks) $^{\mathrm{a}}$ & $29(24-35)$ & $28(25-33)$ \\
Weight at birth (grams) $^{\mathrm{a}}$ & $1475(780-2395)$ & $1089(500-1480)$ \\
\hline
\end{tabular}

a Summary on average (minimum and maximum) of the values gestational age and weight at birth.

software SPSS v 21.0.

\section{Results}

The inclusion criteria were met by 44 infants with an average age of 3 months in infants with PIVH and 2,6 months in infants without PIVH ( $p=0.284$ ), the sample characterization is shown in Table 1 .

As the study comprised preterm infants, the association of risk indicators for hearing loss was observed: Apgar score below 4 in the first minute and/or below 6 in the fifth minute, ICU stay, weight at birth below $1500 \mathrm{~g}$ and use of ototoxic medication. However, there was no significant correlation between the presence of these indicators and the alterations found in the BAEP (Table 2).

Similarly, the difference between male and female in infants in the group with PIVH did not have a confounding effect in the analysis of the BAEP results $(\mathrm{p}=0.467)$.

The classification of the PIVH severity (I, II, III and IV) was performed by the hospital's Neonatology team, with six infants with grade I, seven with grade II, three with grade III and two with grade IV.

In the auditory investigation, the included infants presented TEOAE in both ears, performed before the electrophysiological assessment. In relation to the BAEP results, a significant increase was observed in absolute latency values of waves I, III and V, as well as in interpeak intervals I-III and I-V bilaterally in infants with PIVH (Table 3).

\section{Discussion}

PIVH can lead to serious neurological and sensory sequelae such as: cerebral palsy, visual and auditory impairments, mental retardation, acute intracranial hyperten-sion, hypoxic-ischemic brain injury, posthemorrhagic hydrocephalus and periventricular hemorrhagic infarction [17]. The lower the gestational period and the birth weight, the more frequent the PIVH, due to the immaturity of the central nervous system. Between the 26th and 32 nd weeks of gestation, the period of great proliferation of the germinal matrix, the initial site of PIVH, occurs. After this period, involution of this region occurs and the risks of bleeding decrease, however, due to the difficulty in correctly establishing the gestational age, the birth weight becomes the most commonly variable used to define the risk group for PIVH $[1,2,10]$.

Currently, the incidence of PIVH has decreased and survival rates have increased due to improvements in overall newborn care and technological advancement $[1,9,18]$.

Table 2

Comparison between risk indicators for hearing loss in relation to BAEP latencies.

\begin{tabular}{llll}
\hline Latencies of the right ear & $p$ & Latencies of the left ear & $p$ \\
\hline I & 0,143 & I & 0,861 \\
III & 0,826 & III & 0,965 \\
V & 0,861 & V & 0,826 \\
I-III & 0,421 & I-III & 0,826 \\
III-V & 0,692 & III-V & 0,930 \\
I-V & 0,448 & I-V & 0,759 \\
\hline
\end{tabular}

Mann-Whitney test. 
Table 3

Comparison between absolute and interpeak latencies of BAEP in both ears in infants with and without Peri-Intraventricular Hemorrhage.

\begin{tabular}{|c|c|c|c|c|c|c|c|c|}
\hline \multirow{2}{*}{\multicolumn{2}{|c|}{$\begin{array}{l}\text { Absolute and } \\
\text { interpeak } \\
\text { latencies }\end{array}$}} & \multicolumn{3}{|c|}{ without PIVH $(n=26)$} & \multicolumn{3}{|c|}{ with PIVH $(\mathrm{n}=18)$} & \multirow[t]{2}{*}{$p$} \\
\hline & & Md & Min & $\operatorname{Max}$ & Md & Min & Max & \\
\hline \multirow[t]{6}{*}{$\mathrm{RE}$} & I & 1,43 & 1,27 & 1,67 & 1,57 & 1,44 & 2,04 & $<0.001$ \\
\hline & III & 4,00 & 3,67 & 4,30 & 4,50 & 4,12 & 5,29 & $<0.001$ \\
\hline & $\mathrm{V}$ & 6,33 & 5,80 & 6,73 & 6,77 & 6,20 & 8,13 & $<0.001$ \\
\hline & I-III & 2,57 & 2,00 & 2,93 & 2,84 & 2,50 & 3,70 & $<0.001$ \\
\hline & III-V & 2,27 & 2,03 & 2,80 & 2,34 & 2,00 & 2,94 & 0,383 \\
\hline & $\mathrm{I}-\mathrm{V}$ & 4,88 & 4,47 & 5,33 & 5,19 & 4,68 & 6,54 & 0,003 \\
\hline \multirow[t]{6}{*}{ LE } & I & 1,48 & 1,27 & 1,97 & 1,67 & 1,46 & 1,99 & $<0.001$ \\
\hline & III & 4,07 & 3,77 & 4,57 & 4,49 & 4,07 & 5,16 & $<0.001$ \\
\hline & $\mathrm{V}$ & 6,32 & 5,83 & 6,80 & 6,72 & 6,23 & 8,03 & $<0.001$ \\
\hline & I-III & 2,59 & 2,17 & 3,03 & 2,81 & 2,37 & 3,57 & 0,011 \\
\hline & III-V & 2,30 & 1,93 & 2,90 & 2,28 & 1,90 & 3,15 & 0,550 \\
\hline & I-V & 4,80 & 4,53 & 5,40 & 5,03 & 4,56 & 6,44 & 0,004 \\
\hline
\end{tabular}

Caption: PIVH: Peri-Intraventricular Hemorrhage; RE - right ear; LE - left ear. Mann-Whitney for independent samples.

Descriptive summary in median (minimum and maximum).

The study of the effects of PIVH on hearing is still little explored in the literature, and the BAEP test as an objective measure of functional integrity assessment of the auditory pathway allows to verify the existence of lesions in these structures, possibly caused by PIVH.

Studies show the occurrence of hearing impairment in preterm infants weighing below $1500 \mathrm{~g}$, a characteristic frequently found in infants with PIVH, and their findings reveal the absence of response in electroacoustic tests and different findings at electrophysiological thresholds $[19,20]$. However, the purpose of this study was to verify the neural conduction time of the sound in hearing infants and who suffered from PIVH.

Thus, when comparing the BAEP results in groups of infants with and without PIVH, a significant increase was observed in the absolute latencies of waves I, III and V, as well as in the interpeak intervals I-III and I-V in both ears.

The analysis of the absolute latencies of waves I, III and V, and of the BAEP, allows to verify the time between the stimulus and the formation of the neural activity, its increase is often related to the preneural conditions, such as alterations of middle ear and cochlear hearing loss. However, this study included only infants with present otoacoustic emissions, allowing to demonstrate the occurrence of a synaptic dysfunction in the auditory pathway up to the brainstem in infants with PIVH.

The analysis of the interpeak latencies allows to verify the speed of the connections across the sites generating the auditory neural response. The increase in interpeak latency in I-III and I-V in infants with $\mathrm{PIVH}$ showed that there was impairment in the synaptic conduction of the lower brainstem, that is, the peripheral portion of the auditory pathway.

Similarly, Angrisani et al. [21] reported increased latency of waves III and V, and of interpeak interval I-V in infants who had PIVH, indicating that these individuals are susceptible to retrocochlear impairment.

Another finding to be discussed would be regarding the degree of $\mathrm{PIVH}$, which can range from I to IV. In the present study, there was a higher occurrence of grades I and II, which, despite having a better prognosis due to the higher chances of reabsorption, these findings show that there is synaptic conduction impairment in the auditory pathway in these infants.

Thus, there is a need to monitor the evolution of auditory abilities in order to better understand the effects of PIVH on child development.

\section{Conclusion}

This study demonstrated changes in auditory structures at the level of the lower brainstem in infants who had PIVH regardless of the degree.

\section{Conflicts of interest}

The authors report no conflicts of interest.

\section{Acknowledgements}

We thank the children and their guardians for participating in the research.

\section{References}

[1] S.T. Marba, J.P. Caldas, L.E. Vinagre, M.A. Pessoto, Incidence of periventricular/ intraventricular hemorrhage in very low birth weight infants: a 15-year cohort study, J. Pediatr. 87 (2011) 505-511.

[2] J.J. Volpe, Intracranial hemorrhage: germinal matrixintraventricular hemorrhage of the premature infant, Neurol. N.born 5 (2008) 517-588.

[3] J.P.S. Caldas, C.A. Braghini, T.N. Mazzola, M.M.S. Vilelac, S.T. Marba, Peri-intraventricular hemorrhage and oxidative and inflammatory stress markers in verylow birth weight newborns, J. Pediatr. 91 (2015) 373-379.

[4] J.A. Ferraro, J.D. Durrant, Auditory evoked potentials: overview and basic principals, Handb. Clin. Audiol. 4 (1994) 317-338.

[5] K. Patra, D.W. Costello, G. Taylor, N.M. Minich, M. Hack, Grades I-II intraventricular hemorrhage in extremely low birth weight infants: effects on neurodevelopment, J. Pediatr. 149 (2006) 169-173.

[6] H. Bassan, Intracranial hemorrhage in the preterm infant: understanding it, preventing it, Clin. Perinatol. 36 (2009) 737-762.

[7] L.A. Papile, J. Burstein, R. Burstein, H. Koffler, Incidence and evolution of subependymal and intraventricular hemorrhage: a study of infants with birthweights less than $1500 \mathrm{~g}$, J. Pediatr. 92 (1978) 529-534.

[8] E.H. Roland, A. Hill, Germinal matrix-intraventricular hemorrhage in the premature newborn: management and outcomes, Neurol. Clin. 21 (2003) 833-851.

[9] L.O. Tiensoli, L.M. Goulart, L.M. Resende, E.A. Colosimo, Hearing screening in a public hospital in Belo Horizonte, Minas Gerais State, Brazil: hearing impairment and risk factors in neonates and infants, Cad. Saúde Pública 23 (2007) 1431-1441.

[10] S. Bolisetty, A. Dhawan, M. Abdel-Latif, B. Bajuk, J. Stack, K. Lui, Intraventricular hemorrhage and neurodevelopmental outcomes in extreme preterm infants, Pediatrics 133 (2014) 55-62.

[11] C.C. Stumpf, C. Gambini, L.C.B. Jacob-Corteletti, S.M. Roggia, Newborn hearing screening: a study in Curitiba-PR, Rev. CEFAC 11 (2009) 478-485.

[12] A. Widziszowska, G. Namyslowski, Assessment of hearing organ activity in a group of neonates with central, Int. J. Pediatr. Otorhinolaryngol. 75 (2011) 1280-1284.

[13] A. Yasuhara, Y. Kinoshita, A. Hori, S. Iwase, Y. Kobayashi, Auditory brainstem response in neonates with asphyxia and intracranial haemorrhage, Eur. J. Pediatr. 145 (1986) 347-350.

[14] D.L. Jewett, M.N. Romano, J.S. Williston, Human-auditory evoked potential: possible brain stem components detected on the scalp, Science 167 (1970) 1517-1518.

[15] E.A. Guzman, J.R.D. Bertagnon, Y. Juliano, Frequency of peri-intraventricular hemorrhage and its associated factors in premature newborns, Einstein 8 (2010) 315-319.

[16] Joint Committee on Infant Hearing, Year 2007 position statement: principles and guidelines for early hearing detection and intervention programs, Pediatrics 120 (2007) 898-921.

[17] T.M. O'Shea, E.N. Allred, K.C. Kuban, D. Hirtz, B. Specter, S. Durfee, Intraventricular hemorrhage and developmental outcomes at 24 months of age in extremely preterm infants, J. Child Neurol. 27 (2012) 22-29.

[18] L.A.T. Neves, J.A.C. Neto, D. Kneipp, L.G. Fonseca, M.P. Rosado, M.R.P. Barreto, Intracranial Hemorrhages in Preterm Newborns. Casuistry of the Albert Sabin Hospital's Neonatal Unit. HU Ver, (2007), pp. 47-52.

[19] R.Z. Luo, X.Y. Wang, R.J. Wen, J.L. Zhou, J. Liang, Z.Y. Huang, et al., Evaluation and analysis of auditory function and its clinical characteristics in low birth weight premature infants, Zhonghua er bi yan hou tou jing wai ke za zhi 44 (2009) 893-899.

[20] D.P.C. Silva, P.S. Lopez, J.C. Mantovani, Auditory brainstem response in term and preterm infants with neonatal complications: the importance of the sequential evaluation, Int. Arch. Otorhinolaryngol. 19 (2015) 161-165.

[21] R.G. Angrisani, A.M.C. Fagá, A.L. Goulart, M.F. Azevedo, Intracranial hemorrhage and central auditory disorders in neonates, Rev. CEFAC 18 (2016) 341-347. 